\title{
Analysis and application of steering systems on a steering test bench
}

\author{
Christoph Nippold ${ }^{1} \cdot$ Ferit Küçükay $^{1} \cdot$ Roman Henze $^{1}$
}

Received: 3 December 2015/Accepted: 6 May 2016/Published online: 23 May 2016

(C) Springer International Publishing Switzerland 2016

\begin{abstract}
This paper focuses on the test bench-based application and analysis of electro-mechanical power steering (EPS). In the first part, the setup and physical structure of the test bench are described. It is shown how control parameter changes can be measured, using the assistance amplification as an example. In the next chapter, a method for a test bench-based pre-application is introduced. A hardware change in the servo-unit alters the steering feel and requires compensating that by changing the control parameters of the current (electrical) controller. The method of Design of Experiments is introduced to reduce the number of combinations of the control parameters. Based on the predefined test plan, measurements are made to create an empirical model. This model is further used in an optimisation problem to compute the optimal set of parameters. This optimum is validated by additional measurements.
\end{abstract}

Keywords Steering application - Test bench .

Design of experiments

\section{Introduction}

The steering behaviour has a direct impact on the customers' perception of the vehicle's character. The steering wheel provides information about the driving condition; therefore, the steering system directly impacts the driver's guidance behaviour.

Christoph Nippold

c.nippold@tu-bs.de

1 Institute of Automotive Engineering, TU Braunschweig, Hans-Sommer-Str. 4, 38106 Brunswick, Germany
Developing and applying a brand-specific steering feel is a time-consuming process. A huge benefit of today's electro-mechanical steering systems is the ability to provide steering torque independently of the driver. Thereby, an almost freely defined steering assistance torque can be specified based on a variety of steering functions. However, a variety of steering functions also yields a variety of parameters, which makes the process of tuning very time consuming. Figure 1 shows a schematic overview of the steering functions of an EPS. In general, steering functions can be divided into "steering feel" and "advanced functions" (according to [1, 2, and 3]). Based on the current information of the steering and driving state (sensors), the steering functions determine the amount of steering assistance torque to be provided. The summed forces of the various functions yield a target torque. An in-series connected current controller controls the target torque. The research investigations at the steering bench mainly focus on steering feel functions and designing the current controller which has a significant impact on the steering feel [4].

Figure 2 provides an overview of different application processes of steering systems. Today, this is mainly done iteratively in the vehicle, as shown in the white fields of Fig. 2. The grey fields show the current research topics, making the process of application more efficient in the future. In general, besides real driving, the application engineer has three tools to judge steering behaviour:

1. Simulator

2. HiL test bench

3. Simulation.

A simulator can provide a high amount of realism and actual car parts. In addition, steering functions can be judged directly by a driver. This tool is introduced in [5]. 
Fig. 1 Overview and classification of steering functions

Fig. 2 Variants of the tuning process of steering systems, according to [6]
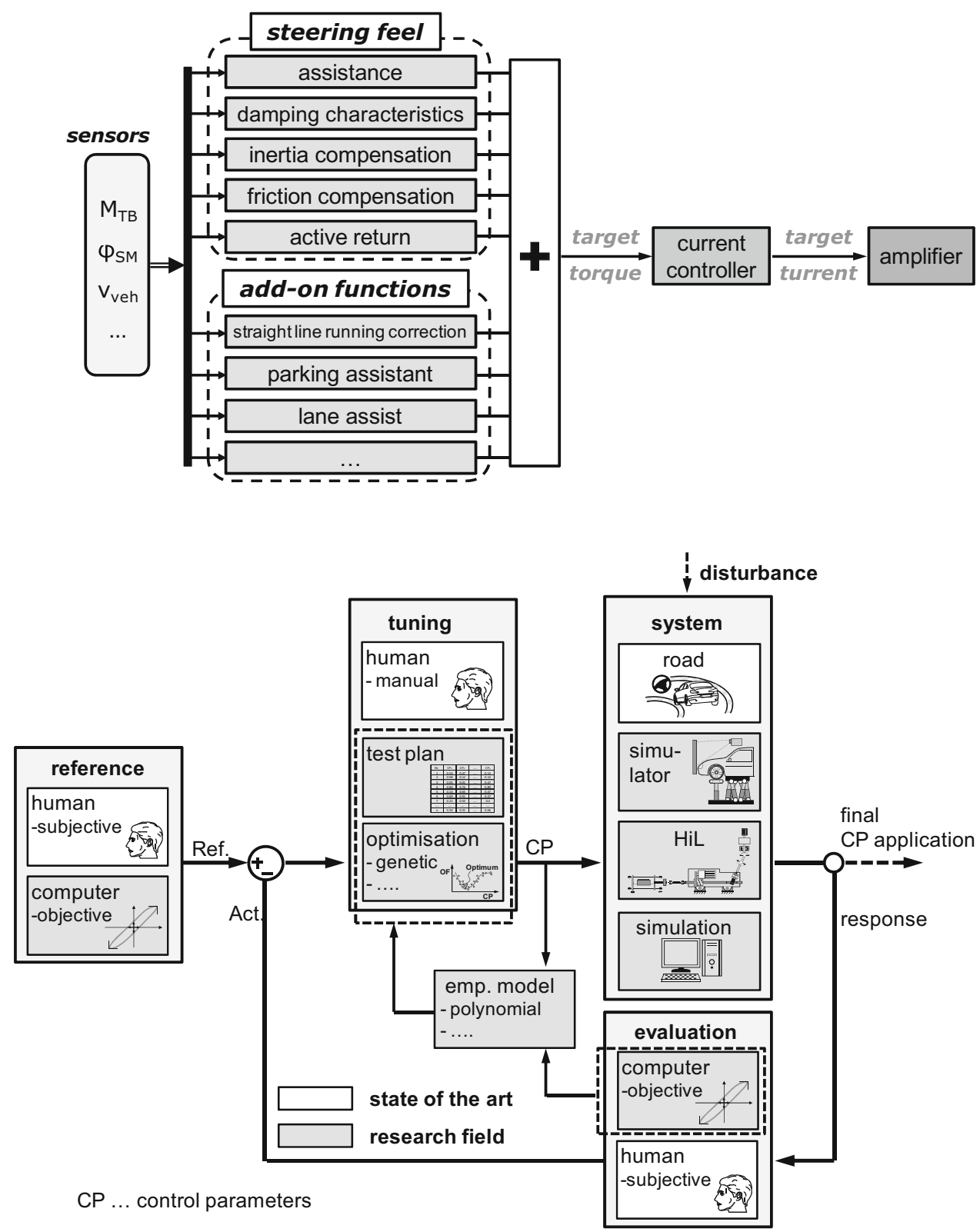

That makes it possible to apply and test steering systems very early in the development process. The next application stage is the HiL test bench, which makes it at least necessary to use the steering gear as a single hardware component. The intermediate steering shaft, track rod, and other hardware components are optional. Lee et al. [7] uses a HiL steering test bench to evaluate the influence of the sensitivity steering functions and the stiffness of the torsion bar on the steering behaviour. Possible cross-influences are also evaluated.

Koegeler et al. [8] introduces a general test bench-based method, using the assistance amplification (ASA) as an example. By taking measurements, a variety of ASA control parameters is tested, which is not feasible in the vehicle in general. Using the method of Design of Experiments (DoE), a model describing the connection between the ASA parameters and the vehicles reaction is derived. Thus, the application engineer gets the possibility to choose a defined pre-application on the computer describing the relation between steering torque and the vehicle's reaction.

The presented work focuses on the detailed pre-application of a steering system at the HiL test bench. Crucial for the quality of the pre-application is the quality of the test bench. In the first part, the setup and precision of the test bench are described and verified using the ASA. In the next step, a test bench-based method to pre-apply steering systems is introduced. Therefore, a practically relevant example is used to show how a steering system is pre- 
applied to defined reference behaviour. The last part of the work gives an outlook on how to analyse and evaluate steering systems at the Institute of Automotive Engineering at the TU Braunschweig.

\section{Steering test bench}

The precision of the actuators (steering angle and counterforce) and the resolution of defined state variables are critical for the quality of a test bench-based pre-application of a steering system. In particular, the ability to measure minimal changes in the application is the basis of a practicable method for the application process at the steering test bench. As a specific target area, the applicable step width of the force of the steering system's servo motor can be named which is at about $2 \mathrm{~N}$ at the steering rack.

Figure 3 shows the schematic setup and the signal flow of the steering test bench. The only installed hardware components to be tested are the steering gear and the intermediate steering shaft. Since the primary focus lies on researching the application of the steering gear, the steering column is intentionally left aside, because the focus of this work is to analyse the difference of two power packs.

The rack force is provided by a linear motor via a modified track rod. This track rod is equipped with two track rod joints to compensate a potential coaxial behaviour of the rack and the linear motor's axle. This setup eliminates restraints, which could otherwise lead to higher friction within the steering gear.

The counterforce of the wheel is provided by a linear motor which has a very good precision (see Fig. 4) and high dynamics. Since the force has to be filtered, there is a phase offset between target and actual force. This offset is at about 4 degrees at $1-\mathrm{Hz}$ excitation, which is relatively low compared to the real-vehicle behaviour (steering wheel angle to lateral acceleration). The choice of a linear motor has been made deliberately to achieve the highest possible accuracy of the force. The maximum possible force of the linear motor is $2500 \mathrm{~N}$, which is sufficient for the investigation of on-centre steering. Using two actuators, the manoeuvres of the driving dynamic linear area (approx. $5 \mathrm{~m} / \mathrm{s}^{2}$ lateral acceleration) can be performed. Thus, it is possible to cover a variety of relevant driving manoeuvres in the first approach. For parking manoeuvres, driving manoeuvres with high lateral accelerations and vehicles with a high front axle load, the actuators have to be modified. It has to be said that for controllers like the used force control in most cases, there is a trade-off between accuracy and maximum forces. This makes it necessary to adapt the control system to the focus of each investigation. The linear motor is equipped with a transducer measuring the current position of the stator. This measured displacement acts as the input for the vehicle model computing the required rack force in real time.

This vehicle model is an advanced single-track model which has been expanded by non-linear tyre behaviour,
Fig. 3 Schematic structure of the steering test rig

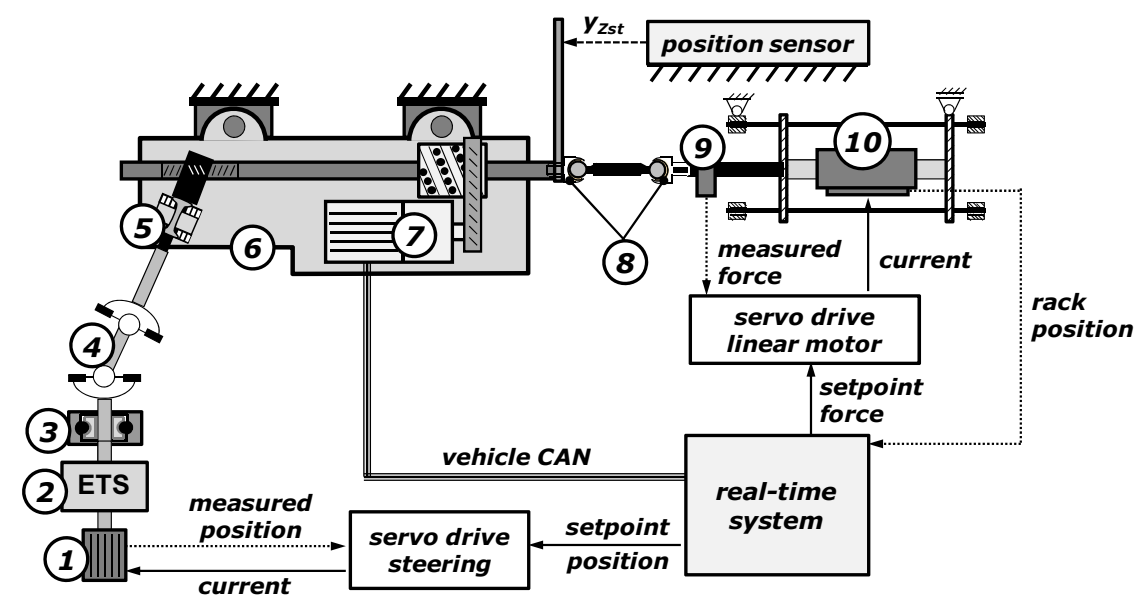

\begin{tabular}{|c|l|c|l|}
\hline 1 & servo motor - steering wheel angle & 6 & steering gear housing \\
\hline 2 & external torque sensor (ETS) & 7 & $\begin{array}{l}\text { power pack: combined unit of } \\
\text { servo motor and ECU }\end{array}$ \\
\hline 3 & bearing & 8 & steering tie tod joint \\
\hline 4 & intermediate steering shaft & 9 & load cell \\
\hline 5 & torsion bar & 10 & linear motor \\
\hline
\end{tabular}


Fig. 4 Control performance of test rig (test frequency: $0.2 \mathrm{~Hz}$ )

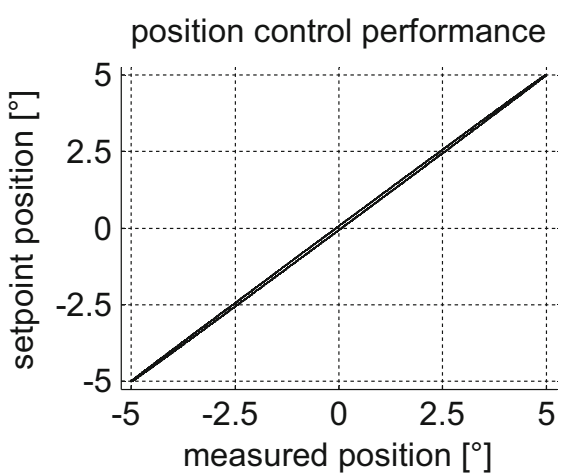

relaxation lengths of the tyre, and suspension elasticity. A more in-depth modelling approach (roll behaviour, camber plates model, spring and damper settings, etc.) is not necessary for the underlying investigations, since an application should match a reference behaviour measured by the test bench, and the investigated driving manoeuvres have a maximum lateral acceleration of approximately $2 \mathrm{~m} / \mathrm{s}^{2}$. However, it is conceivable to add more investigations on the influence of components of the suspension system on the steering feel using a validated more detailed model. Furthermore, the simulated vehicle speed, along with other necessary state variables, is sent to the ECU of the steering in real time via the vehicle CAN.

In addition, the test bench is equipped with external sensors. First, a high precision distance sensor that has a measuring range of $10 \mathrm{~mm}$ with a precision of approx. $0.15 \mu \mathrm{m}$ and which is mounted to the base of the test bench independently. Second, an external torque sensor (see Fig. 3 ETS) between the motor responsible for the steering and the intermediate steering shaft is used. This sensor acts as an additional evaluation criterion and has a precision approximately the same than the torsion bar built in the steering gear.

The ability of the test bench regarding application parameters is shown by changing the assistance amplification (ASA). Figure 5 shows the results of a sine steering $\left(5^{\circ}, 0.2 \mathrm{~Hz}, 100 \mathrm{~km} / \mathrm{h}\right)$ with three variants of the ASA:

- ASA $100 \%$ (basis)

- ASA $100.6 \%$

- ASA $101.2 \%$.

These are the smallest possible changes of the ASA. Each manoeuvre has five periods and is repeated five times, and only the central three periods of each repetition are evaluated. It can be seen that the three variations with different values of the ASA differ significantly in the operating point (see Fig. 6). The dashed lines represent 1 times the standard deviation of the shown average curves. The differences in the application are observable in the torsion bar torque as well as in the rack displacement.
However, the rack displacement shows a higher statistical separability of the application variants, as further shown Fig. 7. Based on a standard deviation, the deviations of the variants are shown for a specific cut (see Fig. 6 A-A and $\mathrm{B}-\mathrm{B})$.

It should be noted at this point that a characteristic point has to be identified for the evaluation and description of steering functions (driving manoeuvres, sensors, and evaluation region) to describe them in the best possible way. This will be confirmed in the next chapter where the application of the current controller significantly differs when building up steering speed or rack displacement.

\section{HiL application}

Developing a method making it possible to fit a steering system application to defined reference behaviour is the goal of this investigation. This is shown using the example of a hardware change of the steering system. Therefore, two power packs (combined unit of servomotor and ECU) with different materials utilized in the electric parts were used. This leads to a different steering feel when used with the same control parameters $(\mathrm{CP})$. The desired steering behaviour (reference behaviour) is achieved by adapting the application of the current controller and comparing the two power packs on the same steering gear to eliminate mechanical influences (see Fig. 8).

A simulative solution to the outlined application would make it necessary to have a detailed model of the steering system's electronic as well as a very complex identification process to determine the influence of the changed material composition. Therefore, a HiL solution is a reasonable compromise between hardware-based complexity and a realistic representation of the influence.

To adapt the steering feel, ten control parameters of the current controller are used. Since the number of available combinations of these parameters is rather high, the method of Design of Experiments (DoE) is used. This minimises the required effort to gain the information needed to 
Fig. 5 Investigation of the steering assistance amplification (ASA)
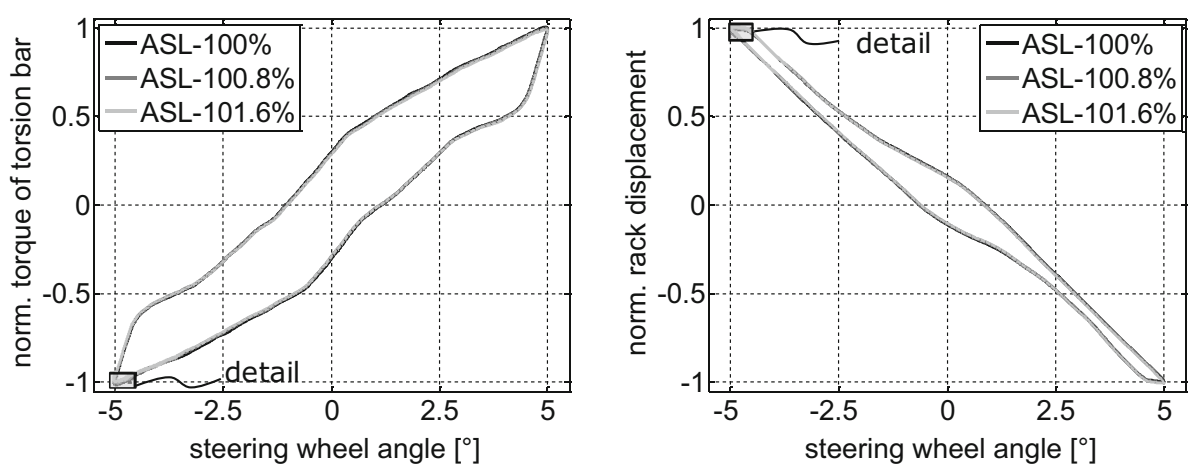

Fig. 6 Investigation of the steering assistance amplification—detail
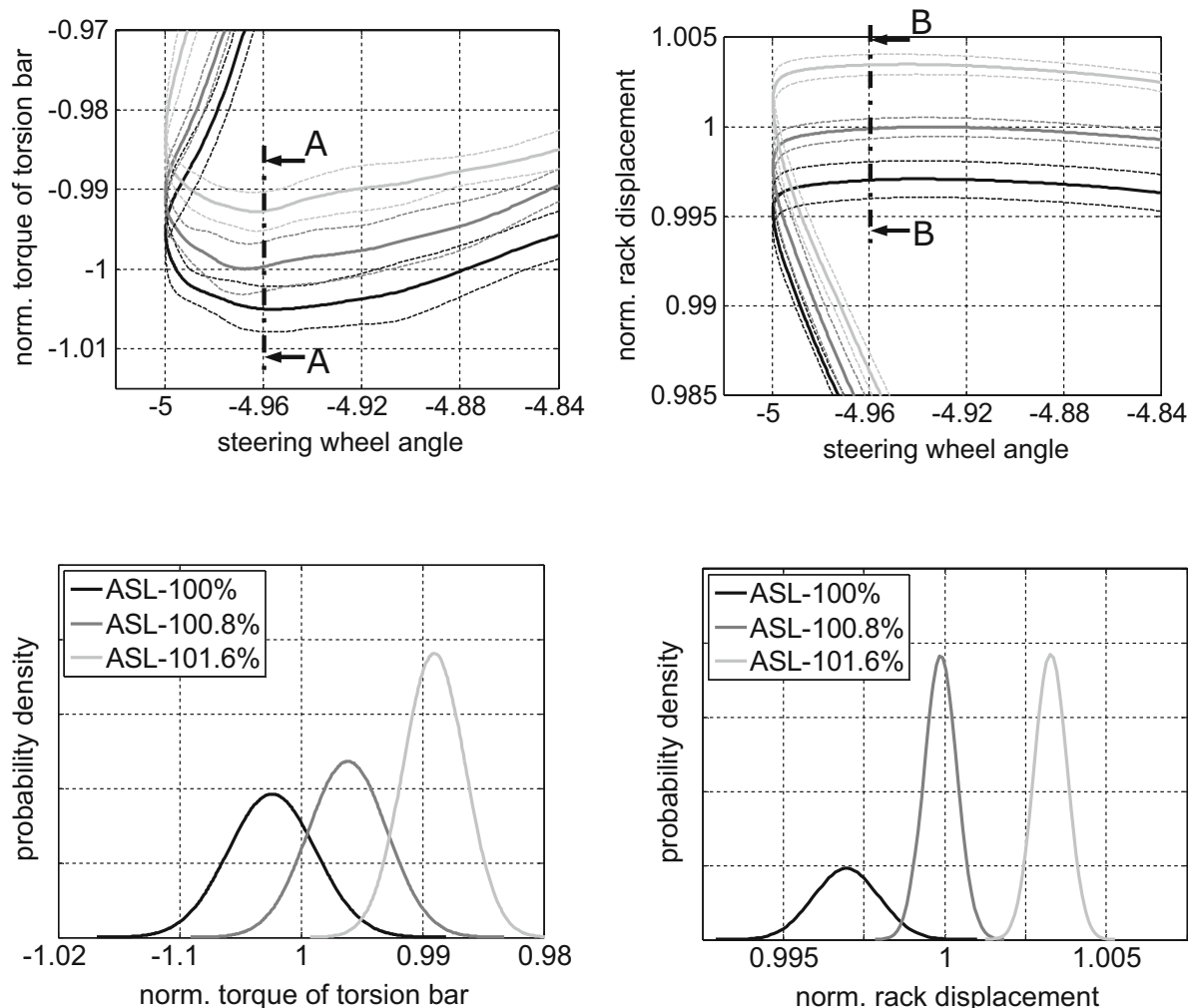

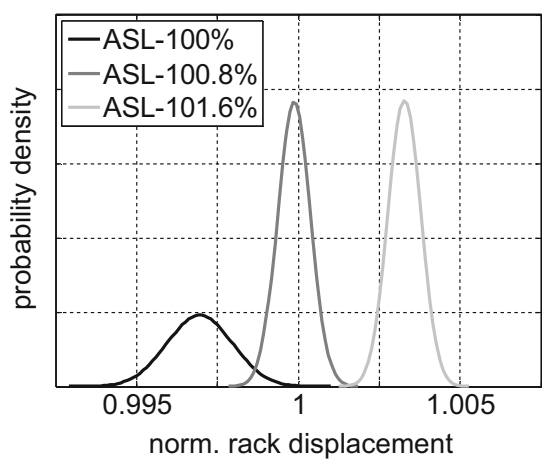

Fig. 7 Investigation of the steering assistance amplification-detailfrequency distribution

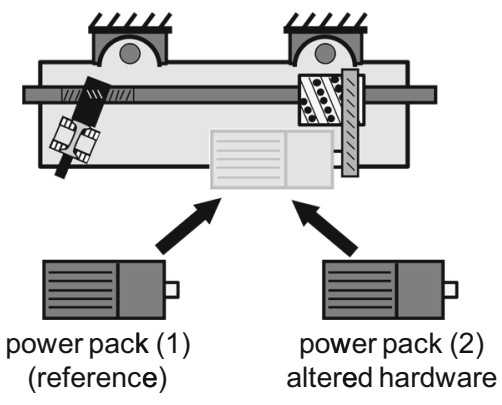

Fig. 8 Investigation of two power packs

describe the system behaviour and the influence of the current controller.

Figure 9 shows the basic scheme of this method for applying a steering system to a reference behaviour using the steering test bench. Based on a defined test plan (1) and subsequent measurements (2), objective data are obtained (3). The system behaviour is computed using a suitable mathematical model afterwards (4). By incorporating the measured behaviour of the reference power pack (0), a parameter set can be obtained in a following optimization process (5). To make sure, this parameter set matches the reference behaviour and compensates the hardware changes, a validation test of the optimised parameters is made.

Altogether, the test bench is significantly less affected by disturbances than real-world testing. Since it is this investigation's aim to measure the slightest changes in the application parameters, various repetitions of the measurements are required to generate a significant objective database. 


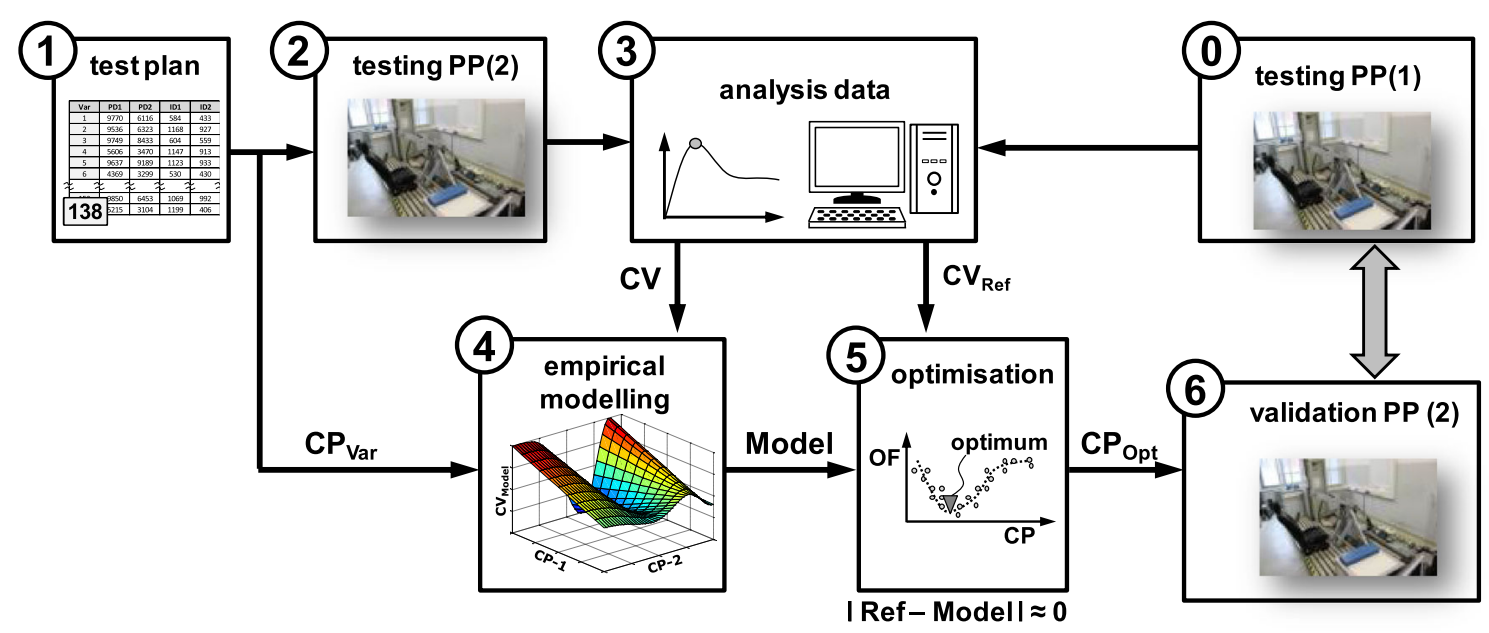

Fig. 9 Method of applying a steering system on a steering test bench to reference behaviour

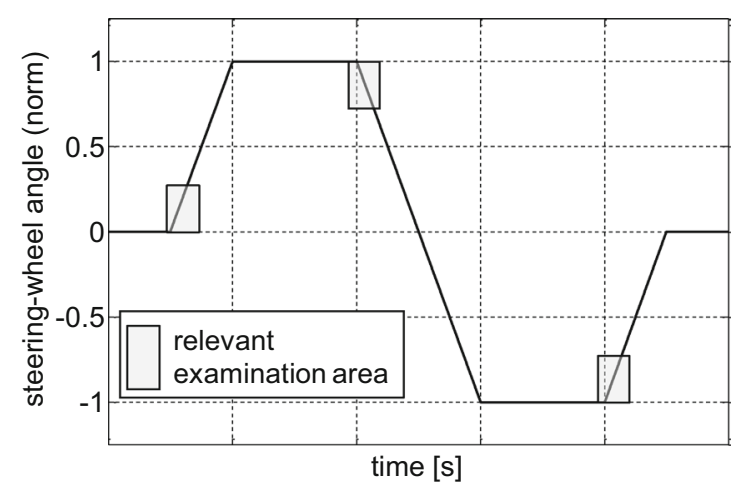

Fig. 10 Characteristic examination areas

First of all, the characteristics are identified to limit the examination area. This is done by performing a sensitivity study. Figure 10 shows the used double trapeze steering manoeuvre in the time domain. The marked areas show characteristic points, where hardware changes lead to significant changes in the steering behaviour (see Fig. 15basis-to-reference line). Particularly, the starting behaviour-starting with $0 \%$ s steering speed-differs between the power packs.

Characteristic values describing the system behaviour properly are identified in this area. The control parameters are optimised by the mathematical model.

\subsection{Test plan and choosing the mathematical model}

As a first step, the generation of a test plan is described, and the decision for a d-optimal plan is justified. Especially, when dealing with experimental results, this method of
DoE is beneficial and, thus, is used in the field of engines and automotive transmissions $[6,9,10,11,[12]$. The generation of the test plan is characterised by minimising the determinant $\left(X^{T} \times X\right)^{-1}$. Here, $X$ is the design matrix describing the configuration space of the control parameters. It is characteristic for a d-optimal plan that the configuration space is predominately filled in the areas close to the border.

Figure 11-left—shows a test plan with a d-optimal setup consisting of two measurement points. The right hand side of that figure shows a space-filling test plan. The measurement uncertainties which are described by the box plot yield a higher gradient of the grey line. This leads to a higher wrapping area around the hatched parts and thus to increasing the variance of the estimated models and their parameters. Using a d-optimal plan, it is possible to estimate the parameters in such a way that the overall model precision increases.

Alongside with the creation of a test plan goes the choice of a mathematical model. In the context of this work, a mathematical model based on polynomial approach is chosen. Equation 1 shows an example of a polynomial model with cross effects.

$y=\underbrace{b_{0}}_{\text {constant/error }}+\underbrace{b_{1} \cdot x_{1}+b_{2} \cdot x_{2}}_{\text {maineffetcs }}+\underbrace{b_{3} \cdot x_{1} \cdot x_{2}}_{\text {interaction }}+\underbrace{b_{4} \cdot x_{1}^{2}+b_{5} \cdot x_{2}^{2}}_{\text {quadraticeffects }}$

This work straitens on a basis function of polynomial degree three. The number of tests is derivable from the number of regressors and the complexity of the model and is stretched by a redundancy factor of 1.6, leading to 138 control parameter variants ([13] recommends a redundancy factor of at least 1.5). 
Fig. 11 Characteristics of d-optimal and space-filling test plan

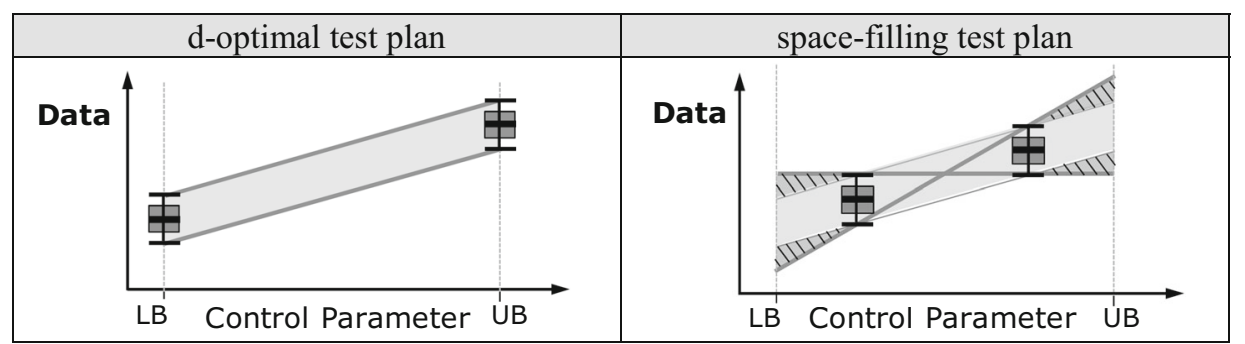

\subsection{Measurements and steering manoeuvres}

All measurements are performed on the respective power pack mounted to the same steering gear. This corresponds with the overview's points 0 and 2 (see Fig. 9). First, the reference unit is measured. To make sure that the reference behaviour is described correctly, the reference unit is measured with three times the amount of repetitions (12 repetitions). Afterwards, the power pack with the changed hardware configuration is mounted, and all measurements (the complete DoE plan) are taken. Aside from the introduced trapezoidal manoeuvre, a sine sweep is performed to detect possible instabilities in the system caused by the current controller's parameters (Fig. 12).

\subsection{DoE and choosing the mathematical model}

After the measurements, the data are processed and the characteristic points are detected. The data processing consists of filtering, selection, outlier detection, and computing of the mean value curves and their standard deviations. Based on the mean value curves, the characteristics are identified. The characteristics of the power packs are identified based on the measurements of the basis and reference behaviour as well as the subjective evaluation. Thereby, the driving behaviour shows that the power packs differ particularly in their start-up behaviour.

Considering, especially, the two external sensor (torque and rack displacement) values observed in objective measurements, significant differences can be seen. Characteristic values are derived from the relation between steering wheel torque and rack displacement to quantify the influence of the changed electronics (see Fig. 13). The low maximum of the steering wheel torque gradient of the basis behaviour reflects the subjective driving impression. To achieve the same steering wheel angle, the driver has to provide a higher steering performance. The two variants differ by $5 \%$ in their maxima. The area between the two curves is used to optimise the basic shape of the steering wheel torque rate.

While (3) describes the reference surface (area underneath the curve of the reference power pack), (4) describes the surface between the reference curve and the curve of the parameter variant (enveloping surface). In the case that area (4) becomes zero, both curves are identical. Figure 13 shows the curves for a steering manoeuvre starting from steering wheel angle equal $0^{\circ}$ (centre position). These characteristic values are computed analogously at the point of operation.

\subsection{Modelling}

Before optimising the control parameters, the mathematical model has to be computed. This is done for each characteristic value (CV) (see Fig. 13. swtp_max_cp Var $_{\text {ar }}$ and A_swtp_cp $\left.p_{\text {Ref-Var }}\right)$. In the course of this, the characteristic values and the control parameters are put into a mathematical relation as seen in Eq. 2.
Fig. 12 Manoeuvres, $C P$ centre position, $O P$ operation point

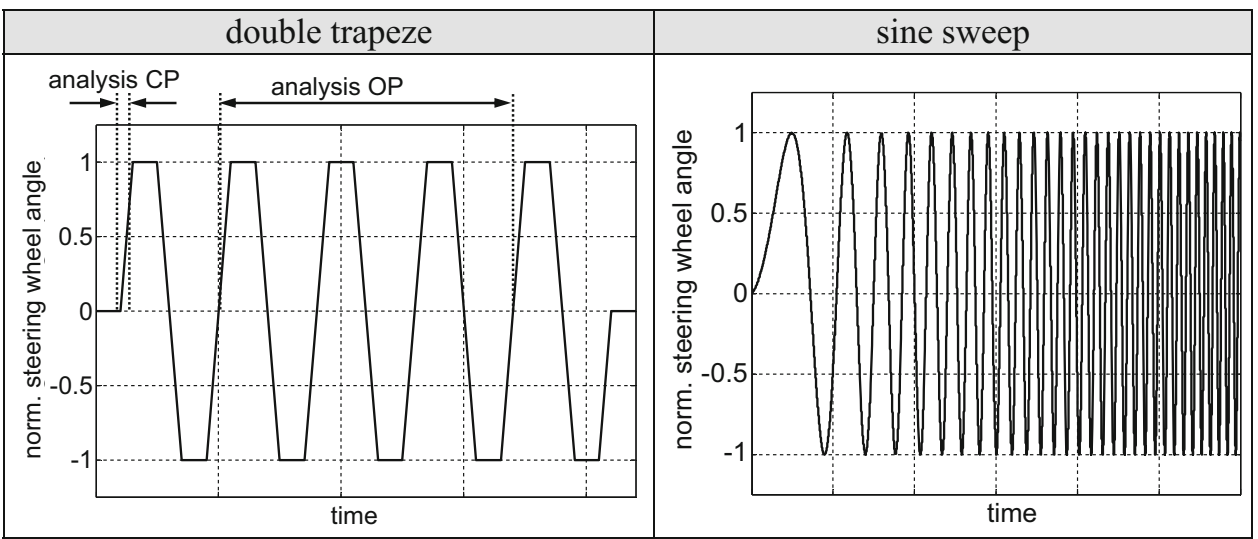


Fig. 13 Identification of characteristic values

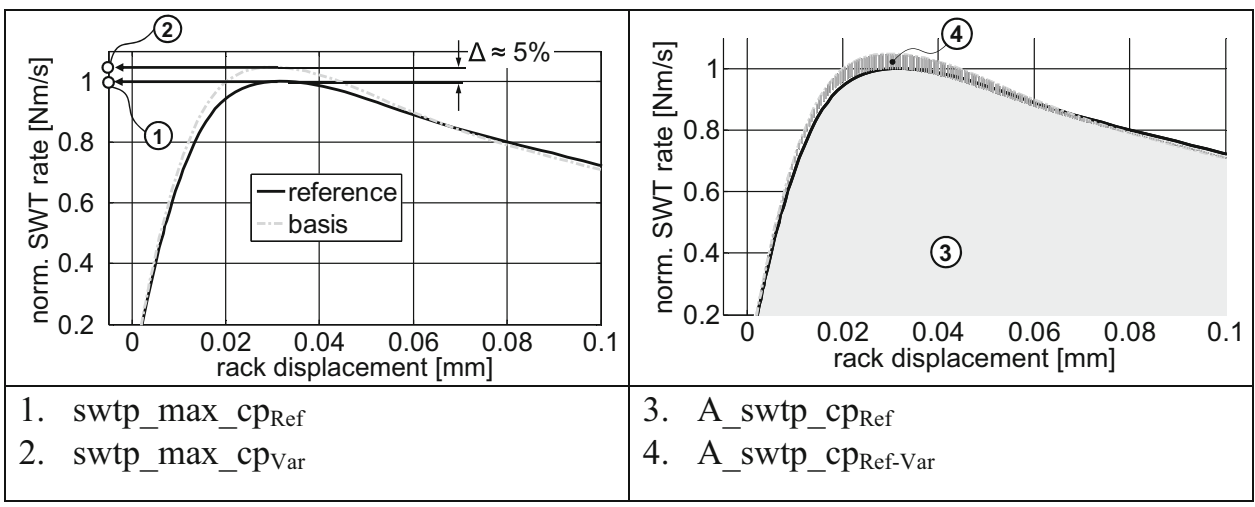

Fig. 14 Model quality

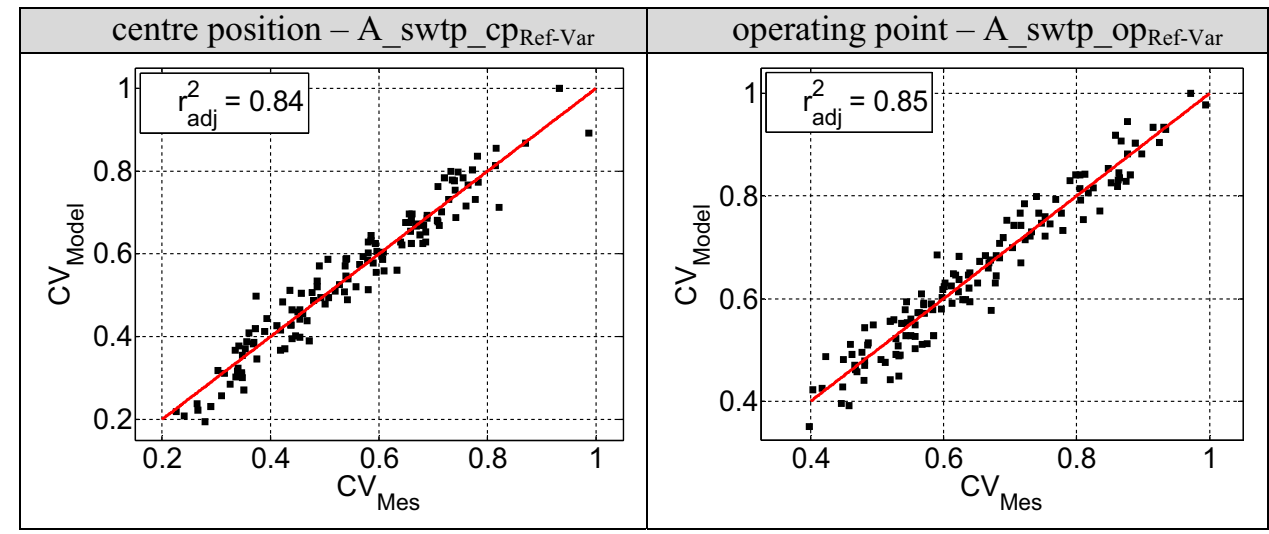

$\mathrm{CV}_{\text {Model }}=f\left(\mathrm{CP}_{1}, \ldots, \mathrm{CP}_{10}\right)$

$\mathrm{CV}_{\text {norm }, j}=\frac{\mathrm{CV}_{\text {Ref }, j}-\mathrm{CV}_{\text {Var }, j}}{\mathrm{CV}_{\text {Ref }, j}}$

Figure 14 shows the quality of the mathematical model for the characteristic values A_swtp_cp Ref-Var $_{\text {and }}$ A_swtp_op $p_{\text {Ref-Var }}$. As a criterion for the quality of the model, the adjusted coefficient of determination, as shown in Eq. 3, is used. There, $n$ is the number of control parameter variants and $p$ is the number of model coefficients.

$r_{\text {adj }}^{2}=1-\frac{\sum_{i=1}^{n} \frac{\left(y_{i}-\hat{y}_{i}\right)^{2}}{n-k-1}}{\sum_{i=1}^{n} \frac{\left(y_{i}-\bar{y}_{i}\right)^{2}}{n-1}}=1-\frac{n-1}{n-p-1} \times\left(1-R^{2}\right)$

The deviation to a more precise model can be explained by measurement uncertainties and the simplified model (polynomial model).

\subsection{Optimisation}

To identify the control parameters describing the reference behaviour ideally, an optimisation process is applied to the obtained mathematical models. To make the various control parameters applicable to an objective function, they are normalised to reference behaviour as described by Eq. 4, with $\mathrm{j}$ being the number of characteristic values to transform for the optimization.
In the case of the $\mathrm{CV}$ describing surfaces between two curves, the normalisation is done by Eq. 5 . Thereby, the area between the reference curve and the parameter variant curve is divided by the surface under the reference curve. The length of examination is described by $z$. Besides, $k$ is the number of the used CV_A, which characterises the enveloping surface.

CV_A $A_{\text {norm }, k}=\frac{\int_{0}^{z}\left|y_{\text {Ref }}(x)-y_{\text {Var }, k}(x)\right| \mathrm{d} x}{\int_{0}^{z} y_{\text {Ref }}(x) \mathrm{d} x}$

Afterwards, the following objective function (OF) is minimised by a genetic optimization algorithm. This type of algorithm uses a method very similar to natural selection. Having starting sets of parameters (randomly generated), it alters the parameters in various ways and only keeps the parameter sets which improved the overall result. After several iterations, an optimum is found; whereby; there is quadratic convergence behaviour. This optimization algorithm is a reliable and robust method for searching solution spaces [14]. The OF consists of the shown characteristic values (see Eq. 6). In principle, it is possible to favour specific areas during the optimization by adding weights. In 
Fig. 15 Validation measurements

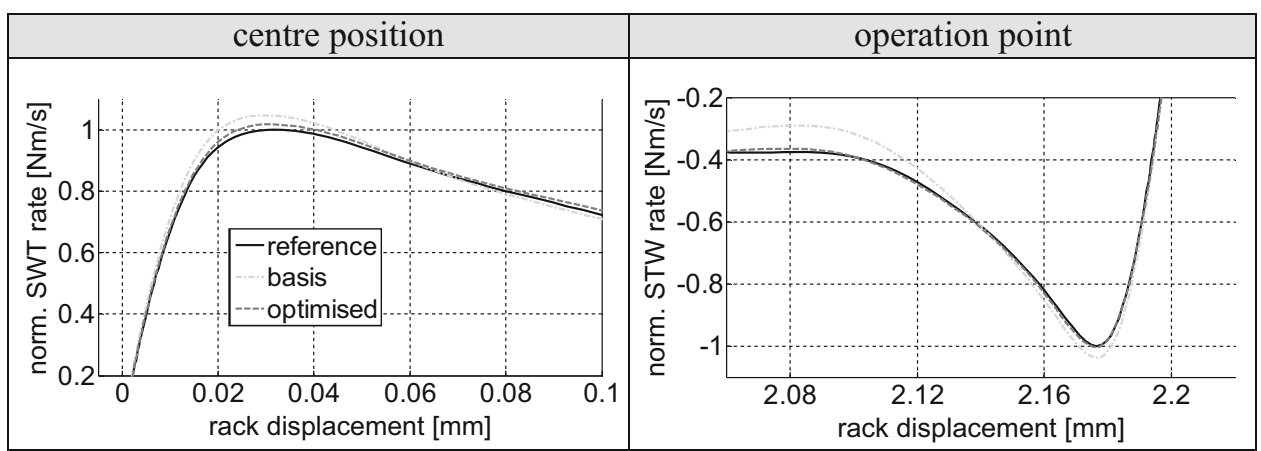

Fig. 16 Improvement of characteristic values by optimisation

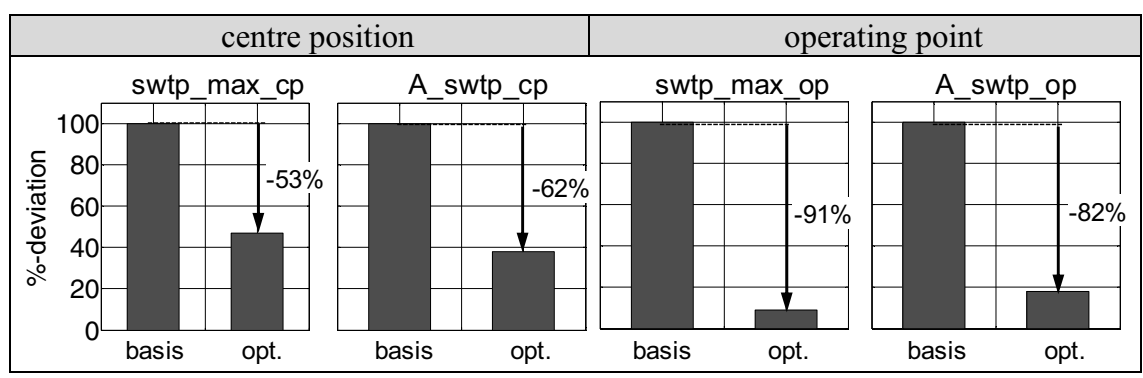

this work, however, steering from the centre position as well as steering back from the operation point has to be optimised equally. Furthermore, the basic shape has to be met in addition to the characteristic maximum. Thus, the optimization is performed without further weights.

$$
\begin{aligned}
\mathrm{OF}= & \min \mid\left(\mathrm{CV}_{\text {norm }, 1}\right)^{2}+\left(C V_{\text {norm }, j}\right)^{2}+\left(C V_{\text {norm }, 1}\right)^{2} \\
& +\left(\mathrm{CV} \_\mathrm{A}_{\text {norm }, k}\right)^{2} \mid
\end{aligned}
$$

\subsection{Validation}

Subsequently, the optimised control parameter set is validated at the steering test bench by applying the parameters to the power pack with the altered hardware and again measuring the power pack. Figure 15 shows the reference behaviour, the basis behaviour, and the behaviour of the optimised parameter set. When considering on-centre steering-Fig. 15 left—-the characteristic maximum of the steering torque rate is lowered closely to the reference behaviour. The characteristic form of the optimised variant is also approaching the reference closely. When steering back from the point of operation, the changes due to the optimization are even more visible. Here, the reference behaviour is met almost completely.

Figure 16 shows the percentage improvement of the optimised parameter set compared to the reference behaviour. Thereby, the $\mathrm{CV}$ maximum and the surface between curves for on-centre steering and back steering from the point of operation are shown. For on-centre steering, an improvement of over $50 \%$ has been achieved, while when steering back from the point of operation, the improvement has been close to $90 \%$. The higher quality regarding the point of operation can be explained by considering the higher number of repetitions (see Sect. 3.2). This yields a more precise identification, especially of the reference behaviour, leading to a more robust overall process.

\section{Dynamic vehicle road simulator}

For future research, a dynamic vehicle road simulator (DVRS) has been developed at the Institute of Automotive Engineering. This research tool is supposed to be used for future development and evaluation of steering functions. In general, this DVRS can be divided into three layers (see Fig. 17). The first layer consists of the hardware elements of the simulator, acting as an interface between driver and simulation environment. The second layer simulates the driving environment with the software SILAB and, therefore, generates scenarios and driving data. The third layer serves as a development environment for applying the implemented steering functions on the simulator, i.e., the developed driver assistance functions are integrated into the simulation environment. Basis of the simulator is a mock-up attached to hexapods. The mock-up is providing real-input elements for controlling the vehicle to the driver. The visualisation of the driving environment is done by the 


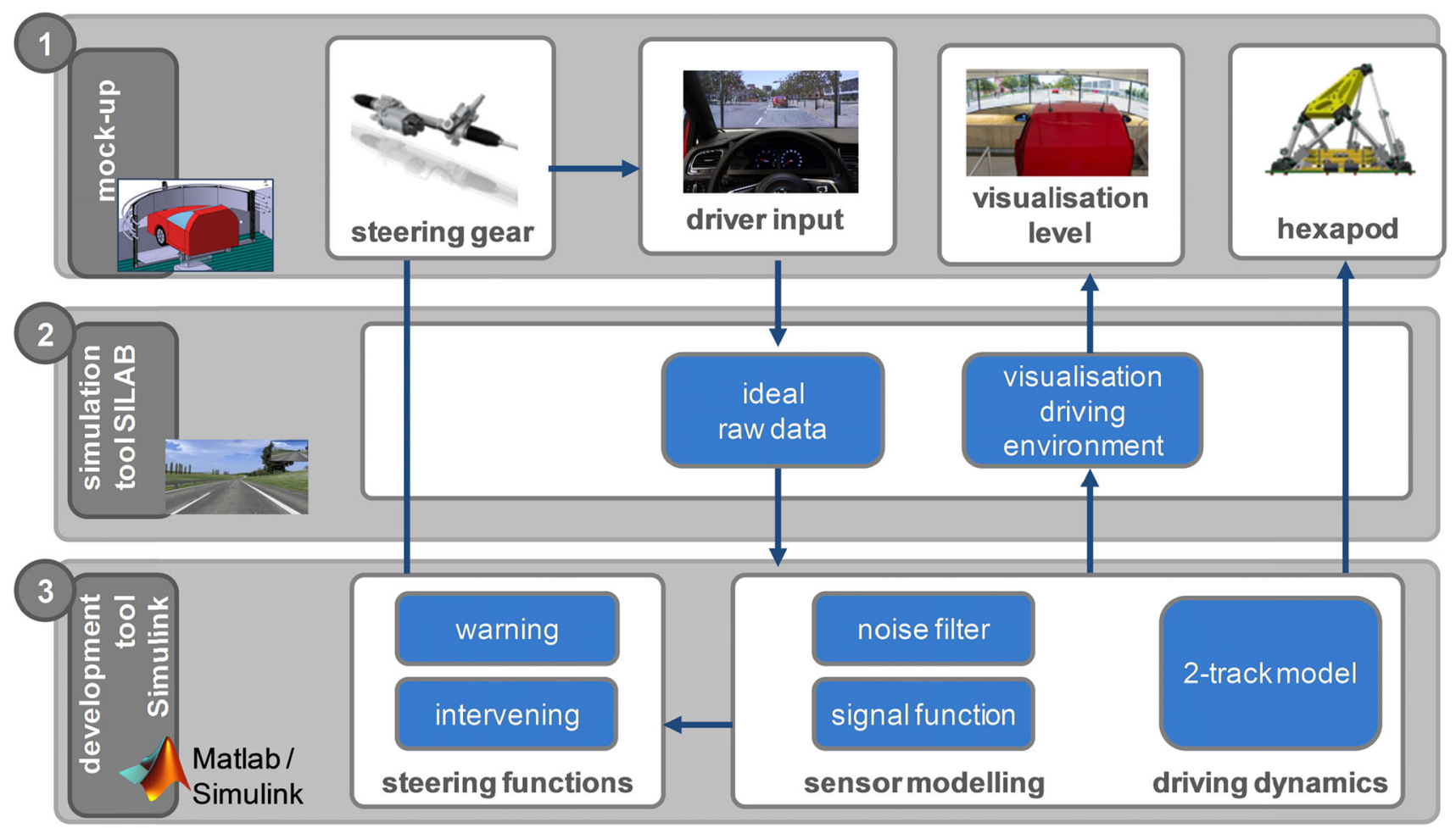

Fig. 17 Development and testing of steering systems using DVRS

simulation software SILAP and displayed on five screens to provide a $180^{\circ}$ view. The hexapods simulate driving dynamics (longitudinal, vertical, and lateral acceleration) of the structure. Simulated state variables based on a validated two-track model as well as simulated sensor values (radar, camera, etc.) serve as inputs to the developed steering functions. This makes it possible to safely develop and evaluate steering functions applicable for automated driving. Therefore, important questions like acceptance studies can be carried out and "handing over" scenarios form automated to manual driving can be developed and tested.

\section{Conclusion and future development}

The presented paper focuses on the test bench-based application of steering systems. In the first step, the qualities of the test bench are evaluated by showing that it is possible to measure minimal changes in the control parameters of the power steering and differentiate them statistically. This forms the basis for a test bench-based application of a steering system. The system behaviour (variation of the control parameters of the current controller) is identified and described with high precision using the method of design of experiments (DoE). In a following optimization process, the ideal control parameters are identified and afterwards validated using the test bench.
This validation shows that a lot of improvements can be made, particularly in the point of operation. The introduced method makes it possible to move the application process from real-world testing to the test bench in the future, since it is especially useful for fitting the steering behaviour to defined reference behaviour.

Furthermore, a dynamic vehicle road simulator is used at the Institute of Automotive Engineering which provides future potential to develop new steering functions efficiently. This makes it possible to generate problem specific scenarios to develop and test new functions, notably in the field of automated driving. In connection with the HiL steering test bench, the new functions can be pre-tuned in detail.

\section{References}

1. Bröcker, M.: New control algorithms for steering feel improvements of an electric powered steering system with belt drive. Veh Syst Dyn Int J Veh Mech Mobil 44(Suppl 1), 759-769 (2006)

2. Brand, W., Brenner, P.: Functional development under conditions similar to series production to optimize the steering feel: $21 \mathrm{st}$ Aachen Colloquium Automobile and Engine Technology, pp 649-660 (2012)

3. Karch, G., Grüner, S.: Mechatronische Lenksysteme (Mechatronic Steering Systems): Band 55, Heft 6: pp 281-289: atAutomatisierungstechnik (2009) 
4. Wimmer, C.: Modellbasierte Entwicklung elektromechanischer Lenkungen: Dissertation, Technische Universität Kaiserslautern. Verlag Dr. Hut, München (2013)

5. Baumann, G., Krantz, W., Pitz, J., Reuer, U., Strecker, J.: Evaluation of steering feeld and vehicle handling in the Stuttgart driving simulator. In: 5th International Munich Chassis Symposium, pp 201-215 (2014)

6. Alvermann, G.: Virtuelle Getriebeabstimmung: Dissertation Technische Universität Braunschweig: Shaker Verlag (2009)

7. Lee, S., Ho Roh, E.: Research on the rig tests for evaluation of steering response of electric power steering(EPS) sub-systems: SAE Technical Paper 2012-01-1920 (2012)

8. Koegeler, H.-M., Schick, B., Pfeffer, P.E., Contini, A., Lugert, M., Schöning, T.: Model-based steering ECU' calibration on a steering-in-the-loop test bench. In: 6th International Munich Chassis Symposium, pp 455-466 (2015)

9. Hagerodt, A.: Automatisierte Optimierung des Schaltkomforts von Automatikgetrieben: Shaker Verlag (2003)
10. Böhl, J.: Effiziente Abstimmung von Automatikgetriebe: Dissertation Technische Universität Braunschweig: Shaker Verlag (2007)

11. Koegeler, H.-M., Fürhapter, A., Mayer, M., Gschweitl, K.: DGIengine calibration, using new methodology with CAMEO: SAE Technical Paper 2001-24-0012 (2001)

12. Isermann, R., Hafner, M., Schüler, M.: Einsatz schneller neuronaler Netze zur modellbasierten Optimierung von Verbrennungsmotoren: Ausgabe 10/2000: pp 704-711: MTZMotortechnische Zeitschrift, Vieweg Verlag (2000)

13. Petersen, H.: Grundlagen der Statistik und der statistischen Versuchsplanung: 4. Ecomed Verlag, Landsberg/Lech, Ergänzungslieferung (1995)

14. Affenzeller, M., Winkler, S., Wagner, S., Beham, A.: Genetic algorithms and genetic programming, modern concepts and practical applications. CRC Press, Boca Raton (2009) 